\title{
A strategy for enhanced tumor targeting of photodynamic therapy based on Escherichia coli-driven drug delivery system
}

\author{
Tao Dai ${ }^{1}$, Farong $\mathrm{Ye}^{2}$, Ping $\mathrm{Hu}^{1,2}$, Xiaohong Pan ${ }^{1}$, Jincan Chen ${ }^{1,5}$, Yunmei Huang ${ }^{3}$, \\ Huanhuan Wang ${ }^{1,5}$, Lei Zhang ${ }^{1,5}$, Mingdong Huang ${ }^{2}$, Jianyong Liu ${ }^{2}$, Jianqiang Su ${ }^{4}$, \\ Xueyuan Chen ${ }^{1,5}$ and Zhuo Chen ${ }^{1,5^{*}}$
}

\begin{abstract}
Escherichia coli (E. coli) DH5a has been recognized as a non-pathogenic bacterial strain with tumor colonization ability. However, whether such a bacteria-driven drug-delivery system can improve the targeting of tumor therapy or not remains essentially untouched. Herein, a series of zinc phthalocyanine $(\mathrm{ZnPc})$ photosensitizers with different numbers of charges were prepared and their electrostatic adhesion properties on $E$. coli were investigated via measuring their fluorescence intensities by flow cytometer. Among these $\mathrm{ZnPc}$ photosensitizers investigated, the ZnPc conjugate with four positive charges (named ZnPc-IR710) exhibited the highest loading capacity and the best fluorescence imaging performance of E. coli. With the help of E. coli, E. coli@ZnPcIR710 presented a significantly enhanced cytotoxicity on human breast cancer MCF-7 cells compared with ZnPc-IR710 (survival rate of tumor cells was $39 \% v$ s. $57 \%$ at a concentration of $50 \mathrm{nmol} \mathrm{L}^{-1}$ ). Moreover, in vivo study showed that E. coli@ZnPc-IR710 remarkably inhibited the tumor growth and resulted in a complete tumor growth suppress in subcutaneous mouse 4T1 breast tumor model. These results demonstrated the great promise of bacterial-guided photodynamic therapy (PDT) in the treatment of solid tumors, and provide a unique strategy to enhance the antitumor efficacy of PDT by utilizing bacterial vectors in tumors.
\end{abstract}

Keywords: photosensitizer, zinc phthalocyanine, bacteria-driven drug delivery system, E. coli DH5a, fluorescent molecular tomography

\section{INTRODUCTION}

In the last few decades, photodynamic therapy (PDT), also called photochemotherapy, has been recognized as a promising cancer therapeutic modality for a number of solid tumors with minimal invasiveness and toxicity, using light, a photosensitizer and oxygen, to create a photochemical reaction and ultimately ablate tumor cells [1-3]. Other attractive features of PDT include reduced need for delicate surgery and lengthy recuperation, reduced scar tissue and defect formation, and short treatment time that is often performed as an outpatient procedure. However, photosensitizers are hard to accumulate in tumor sites, making PDT not yet widely accepted as a frontline cancer therapy.

Recently, more and more attention has been paid to non-pathogenic bacteria in cancer treatment. Some bacteria, including Salmonella [4], Escherichia coli (E. Coli) [5], Bifidobacterium [6] and Clostridium [7], have been reported to be capable of colonizing tumors. Their preferential aggregation in tumors is attributed to immune escape of tumors and the suitable survival conditions of tumor microenvironment (TME), such as weak acidity and hypoxia [8-11]. Meanwhile, the flagellum increases the activity of bacteria penetrating tumor tissue. Therefore, these bacteria are considered to be natural carriers of tumor-targeting drugs $[12,13]$. For instance, mutation of msbB gene in Salmonella VNP20009 results in blockage of lipid A synthesis in lipopolysaccharide (LPS) [14], and

\footnotetext{
${ }^{1}$ State Key Laboratory of Structural Chemistry, CAS Key Laboratory of Design and Assembly of Functional Nanostructures, Fujian Institute of Research on the Structure of Matter, Chinese Academy of Sciences, Fuzhou 350002, China

${ }^{2}$ College of Chemistry, Fuzhou University, Fuzhou 350116, China

${ }^{3}$ Academy of Integrative Medicine, Fujian University of Traditional Chinese Medicine, Fuzhou 350122, China

${ }^{4}$ Key Lab of Urban Environment and Health, Institute of Urban Environment, Chinese Academy of Sciences, Xiamen 361021, China

${ }^{5}$ University of Chinese Academy of Sciences, Beijing 100049, China

${ }^{*}$ Corresponding author (email: zchen@fjirsm.ac.cn)
} 
thus decreases the ability to induce tumor necrosis factoralpha (TNF- $\alpha$ ), which greatly reduces the occurrence of sepsis [15]. However, mice did not tolerate more than $10^{8} \mathrm{CFU}$ of Salmonella, which was far from enough to carry drugs to treat tumors.

To this regard, we developed a high-efficiency bacterium-driven drug delivery system for PDT by employing E. coli DH5a, a non-pathogenic bacterial strain with tumor colonizing ability, as a vector for phthalocyanine (Pc)-based photosensitizer. The reason why we chose $E$. coli as the carrier of the photosensitizer is that the toxicity of $E$. coli is very low $[16,17]$, and thus the mice have strong tolerance to E. coli. In-situ Pc imaging was used to study the aggregation of Pc-carrying bacteria in tumors of tumor-bearing mice. Our results showed that E. coli as a PDT carrier has a good killing effect on hypoxic and nodule core cells, and may significantly improve the therapeutic effect of PDT. These findings demonstrate for the first time the great potential of bacterial-guided PDT in the treatment of solid tumors, and provide a unique strategy to enhance the antitumor efficacy of PDT by using bacterial vectors in tumors.

\section{EXPERIMENTAL SECTION}

\section{Materials and light source}

All reagents were used as received without any further purification. 1,4-Dipegylated phthalocyanine zinc (ZnPc$\left.(\alpha-O H)_{2}\right)$ was synthesized as described previously $[18,19]$. A light-emitting diode (LED) lamp $(\lambda=640-720 \mathrm{~nm}$, $200 \mathrm{~mW}$ ) was obtained from Luma Care Medical Group (USA) and a planar LED light source was customized by Uniglory Electronics (HK) Co. Ltd., which consisted of 24 red light-emitting lamps $(\lambda=680 \mathrm{~nm})$ with a bandwidth of $25 \mathrm{~nm}$ and a dose of $2.4 \mathrm{~J} \mathrm{~cm}^{-2}$ in $1 \mathrm{~min}$.

\section{Cell line, bacterial strain and animal}

Cell lines (human breast adenocarcinoma cell line MCF-7 and mouse mammary carcinoma cell line 4T1) were purchased from Shanghai Institute of Cell Biology, Chinese Academy of Sciences, and maintained in Dulbecco's modified Eagle's medium (DMEM) supplemented with $10 \%$ fetal bovine serum (FBS; Gibco, Invitrogen, USA), $100 \mu \mathrm{g} \mathrm{mL}^{-1}$ penicillin and $100 \mu \mathrm{g} \mathrm{mL}^{-1}$ streptomycin (Gibco, Invitrogen, USA) at $37^{\circ} \mathrm{C}$ with $5 \% \mathrm{CO}_{2}$.

Bioluminescent $E$. coli DH5 $\alpha$ transformed with plasmid pAKlux2.1 $[20,21]$ was used as a model organism in this study. This luminescent strain was cultured in a LuriaBertani (LB) medium (1\% tryptone, $0.5 \%$ yeast extract, $1 \% \mathrm{NaCl}, \mathrm{pH} 7.0)$ at $37^{\circ} \mathrm{C}$.
Kunming mice were purchased from Shanghai SLAC Laboratory Animal Co. Ltd. (China) and were maintained and handled in accordance with the recommendations of Fujian University of Traditional Chinese Medicine institutional animal care and use committee (IACUC). The animals were allowed free access to water and food throughout the course of experiments.

\section{Syntheses of $\mathrm{ZnPc}$ conjugates 1-7}

A series of zinc phthalocyanine ( $\mathrm{ZnPc})$ conjugates (1-7, the structural diagram is shown in Fig. 1) were prepared. Among them, monosubstituted $\beta$-carboxy phthalocyanine zinc ( $\mathrm{ZnPc}-\mathrm{COOH}, 6)$ and 1,4-dipegylated phthalocyanine zinc $\left(\mathrm{ZnPc}-(\alpha-\mathrm{OH})_{2}, 7\right)$ were synthesized as described previously $[18,19]$. The synthetic methods of other photosensitizers (1-5) and the characterization of $\mathrm{ZnPc}$ conjugate 5 (i.e., ZnPc-IR710), including fluorescence excitation spectra $\left(\lambda_{\mathrm{ex}}=610 \mathrm{~nm}, \lambda_{\mathrm{em}}=650-800 \mathrm{~nm}\right)$ are detailed in the Supplementary information.

\section{Detection of singlet oxygen production}

The singlet oxygen production of $\mathrm{ZnPc}$ conjugates 1-7 were measured by using diphenylisobenzofuran (DPBF), where DPBF $\left(240 \mu \mathrm{mol} \mathrm{L}^{-1}\right)$ dissolved in $N, N$ dimethylformamide (DMF) was mixed with suspensions of $\mathrm{ZnPc}$ conjugates 1-7 (at the same concentration of $1 \mu \mathrm{mol} \mathrm{L}{ }^{-1}$ ). The mixture was then irradiated with light of $680 \mathrm{~nm}$ for different time intervals (ranging from 0 to $100 \mathrm{~s}$ ). The absorbance of DPBF at $415 \mathrm{~nm}$ was determined every $10 \mathrm{~s}$ under the light of $680 \mathrm{~nm}$.

\section{Comparison of loading capacity of $\mathrm{ZnPc}$ conjugates 1-7 on E. coli}

The loading capacity of $\mathrm{ZnPc}$ conjugates on bioluminescent $E$. coli was investigated by fluorescence intensity detection of $\mathrm{ZnPc}$ conjugate@E. coli by flow cytometer fluorescence $\left(\lambda_{\mathrm{ex}}=633 \mathrm{~nm}, \lambda_{\mathrm{em}}=675-725 \mathrm{~nm}\right)$ and confocal laser scanning microscopy (CLSM) $\left(\lambda_{\mathrm{ex}}=641 \mathrm{~nm}, \lambda_{\mathrm{em}}=\right.$ $690 \mathrm{~nm})$. In brief, bioluminescent $E$. coli $\left(1 \times 10^{8} \mathrm{CFU}\right)$ was mixed with $\mathrm{ZnPc}$ conjugates 1-7 $\left(10 \mu \mathrm{mol} \mathrm{L}^{-1}\right)$ respectively in phosphate-buffered saline (PBS) solution and incubated with agitation $\left(150 \mathrm{r} \mathrm{min}^{-1}\right)$ at $37^{\circ} \mathrm{C}$ for $10 \mathrm{~min}$. After being centrifuged $\left(4000 \mathrm{r} \mathrm{min}^{-1}\right)$ and then resuspended by PBS, the fluorescence of the suspensions (ZnPc conjugate@E. coli) was analyzed by a CytoFLEX flow cytometer (Beckman Coulter, USA) and imaged by CLSM (Olympus FluoView ${ }^{\text {TM }}$ FV1000, Japan). Furthermore, the kinetic study of $\mathrm{ZnPc}$ conjugate 5 (i.e., ZnPcIR710) on E. coli was carried out with different incubation time periods ( $0,4,8$ and $12 \mathrm{~min}$, respectively) by CLSM. 

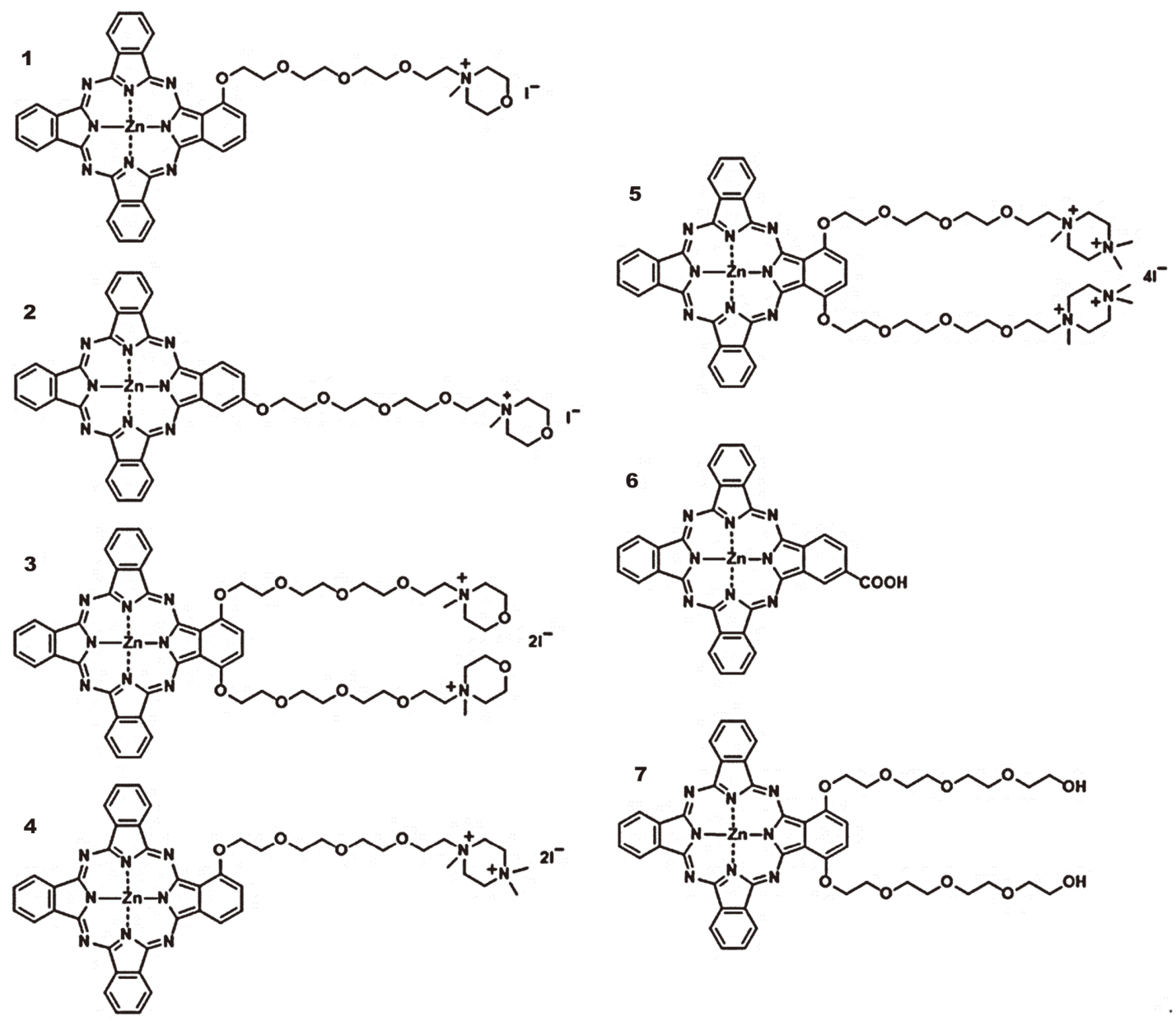

Figure 1 Structural schematic diagram of $\mathrm{ZnPc}$ conjugates 1-7.

Furthermore, the loading capacity of $\mathrm{ZnPc}$ conjugate $\mathbf{5}$ on E. coli was investigated after 10-min incubation with different concentrations of ZnPc-IR710 (1, 2.5, 5, 7.5, 10, 15 and $20 \mu \mathrm{mol} \mathrm{L}^{-1}$, respectively). Then they were centrifuged $\left(4000 \mathrm{r} \mathrm{min}^{-1}\right)$ and washed with PBS for three times. By using sodium dodecyl sulfate (SDS) to dissolve and disperse the sediment, the fluorescence of $\mathrm{ZnPc}$ IR710 was detected by a microplate reader.

Meanwhile, the dark toxicity of ZnPc-IR710 $\left(10 \mu \mathrm{mol} \mathrm{L}^{-1}\right)$ on $E$. coli was evaluated by measuring the optical density at $600 \mathrm{~nm}\left(\mathrm{OD}_{600}\right)$ of E. coli after incubation with E. coli $\left(1 \times 10^{9} \mathrm{CFU}\right)$ for different time periods $(0,1,2,3,4$ and $5 \mathrm{~h}$, respectively).

\section{Cellular uptake of $E$. coli@ZnPc-IR710}

The flow cytometry-based detection of the cellular uptake of E. coli@ZnPc-IR710 was performed on MCF-7 cells $\left(1 \times 10^{5} \mathrm{~mL}^{-1}, 2 \mathrm{~mL}\right)$ which were incubated at $37^{\circ} \mathrm{C}$ for $18 \mathrm{~h}$ to allow the cell adhesion. Typically, adhesive MCF-7

cells were cultured with E. coli@ZnPc-IR710 or ZnPcIR710 (both at a concentration of $100 \mathrm{nmol} \mathrm{L}^{-1}$ ) for $30 \mathrm{~min}$ and analyzed by flow cytometry $\left(\lambda_{\mathrm{ex}}=641 \mathrm{~nm}\right.$, $\lambda_{\text {em }}=675-725 \mathrm{~nm}$ ).

Meanwhile, MCF-7 cells $\left(5 \times 10^{4} \mathrm{~mL}^{-1}, 2 \mathrm{~mL}\right)$ were plated onto a confocal chamber slide and incubated at $37^{\circ} \mathrm{C}$ for $18 \mathrm{~h}$ to allow the cell adhesion. After 30-min incubation with E. coli@ZnPc-IR710 or ZnPc-IR710 (both at a concentration of $100 \mathrm{nmol} \mathrm{L}^{-1}$ ), the slide was gently rinsed with PBS for six times to remove unbounded E. coli@ZnPc-IR710 or ZnPc-IR710 and then mounted for direct confocal microscopic observation (E. coli: $\lambda_{\mathrm{ex}}=488 \mathrm{~nm}$, $\lambda_{\mathrm{em}}=525 \mathrm{~nm}$; ZnPc-IR710: $\lambda_{\mathrm{ex}}=641 \mathrm{~nm}, \lambda_{\mathrm{em}}=690 \mathrm{~nm}$ ).

\section{Photocytotoxicity of E. coli@ZnPc-IR710 in vitro}

Human breast adenocarcinoma MCF-7 cells were seeded into 24-well microplates at $1 \times 10^{5}$ cells per well. Eighteen hours later, these adhesive cells were incubated with E. coli@ZnPc-IR710 or ZnPc-IR710 at various con- 
centrations $\left(0,20,50,100,200,400 \mathrm{nmol} \mathrm{L}^{-1}\right)$. The background control wells in the plate were filled only with culture medium. After incubation for $30 \mathrm{~min}$, the cells were washed with sterile PBS before fresh medium was added. The plates were then illuminated with a light dosage of $9.0 \mathrm{~J} \mathrm{~cm}^{-2}$ by using a planar LED light and then returned to the incubator. After another 24-h incubation, cell survival was assessed by 3-(4,5-cimethylthiazol-2-yl)2,5-diphenyl tetrazolium bromide (MTT) assay.

To further investigate the photodynamic antitumor mechanism of E. coli@ZnPc-IR710 against MCF-7 cells, a double stain apoptosis detection kit containing Hoechst 33342 (a kind of blue-fluorescence dye that stains the condensed chromatin in apoptotic cells more brightly than the chromatin in normal cells) and propidium iodide (PI, a red-fluorescence dye which is excluded by an intact membrane; Beyotime Biotechnology, China) was applied to distinguish normal, apoptotic and dead cell populations. In brief, adhesive MCF-7 cells were incubated with E. coli@ZnPc-IR710 or ZnPc-IR710 (both at a concentration of $100 \mathrm{nmol} \mathrm{L}^{-1}$ ) for $30 \mathrm{~min}$ before exposure to light $\left(3.0 \mathrm{~J} \mathrm{~cm}^{-2}\right)$. Cells were incubated with Hoechst 33342 and PI for $20 \mathrm{~min}$ and then analyzed by flow cytometry.

\section{Establishment of 4T1 tumor-bearing mouse model}

To establish a tumor-bearing mouse model, mouse mammary carcinoma $4 \mathrm{~T} 1$ cells were suspended in sterilized $0.9 \%$ saline solution (cell concentration was adjusted to $1.0 \times 10^{7} \mathrm{~mL}^{-1}$ ), and 0.2 aliquots were subcutaneously inoculated into the right hind flank of the mice. The experiments began when the diameter and thickness of tumors reached $4-8$ and 2-4 mm, respectively, usually 5-7 d after inoculation.

\section{Photodynamic antitumor activity of E. coli@ZnPc-IR710 in vivo}

The antitumor activity of E. coli@ZnPc-IR710 against 4T1 tumor-bearing mouse model was evaluated by growth inhibition analysis. Typically, 4T1 tumor-bearing mice were randomly divided into five groups treated with $0.9 \%$ saline, E. coli $\left(1 \times 10^{9} \mathrm{CFU} \mathrm{mL}^{-1}\right)$, E. coli@ZnPc-IR710 (1× $10^{9} \mathrm{CFU} \mathrm{mL}^{-1}$ containing $2 \mu \mathrm{g} \mathrm{mL}^{-1} \mathrm{ZnPc}$-IR710) and ZnPc-IR710 (2 and $20 \mu \mathrm{g} \mathrm{mL}^{-1}, 10 \mathrm{~mL} \mathrm{~kg}^{-1}$ body weight), respectively. Each group contained eight mice. $24 \mathrm{~h}$ after the treatment, all tumor regions in the mice were irradiated with an LED lamp $(\lambda 640-720 \mathrm{~nm}, 200 \mathrm{~mW}$ for 3 min to receive a total fluence of $30 \mathrm{~J} \mathrm{~cm}^{-2}$ ). To minimize the interference from hair on the tumor surface, the tumor regions were shaved prior to the light irradiation.
Body weights of the mice were monitored daily and the tumor size were measured with a caliper throughout the experiment and calculated by using the ellipsoid volume formula of $W^{2} \times L \times \pi / 6$, where $W$ and $L$ are the width and length of the tumors, respectively. Upon completion of the 7-day treatment, the mice were sacrificed. The tumors were carefully dissected out and weighed. It is worth noting that the dose of $E$. coli used in this study was controlled within the tolerance range of mice to the bacteria, which was determined by injecting different doses of E. coli (from $10^{7}$ to $10^{10} \mathrm{CFU} \mathrm{mL}^{-1}, 100 \mu \mathrm{L}$ ) and monitoring the mice, including daily weight checks.

Meanwhile, the pathologic changes of important organs (heart, kidney, spleen, lung and liver) from the mice treated withE.coli@ZnPc-IR710 were inspected by routine tissue slice with hematoxylin and eosin (H\&E) staining.

\section{Fluorescent molecular tomography imaging}

The 4T1 tumor-bearing mice with approximately equivalent tumor size $\left(\sim 50 \mathrm{~mm}^{3}\right)$ and body weight $(\sim 23 \mathrm{~g})$ were injected intravenously through caudal vein with $200 \mu \mathrm{L}$ of E. coli $\left(1 \times 10^{9} \mathrm{CFU} \mathrm{mL}^{-1}\right)$, E. coli@ZnPcIR710 $\left(1 \times 10^{9} \mathrm{CFU} \mathrm{mL}^{-1}\right.$ containing $2 \mu \mathrm{g} \mathrm{mL}^{-1} \mathrm{ZnPc}-$ IR710), low or high dosage of ZnPc-IR710 (20 and $200 \mu \mathrm{g} \mathrm{kg}^{-1}$ body weight), respectively. Three hours later, the mice were anesthetized with inhaled isoflurane and secured in an cassette to be imaged by a small animal fluorescence molecular tomography (FMT) imaging system (PerkinElmer ViseEn FMT 2500 ${ }^{\mathrm{TM}}$ LX, USA). All FMT scans were carried out by using a 680-nm laser diode with 50-60 source locations and default scan parameters $(\sim 10,000$ counts per pixel and $4.5 \mathrm{~mm}$ spacing between adjacent source locations). The tumor area from the mice receiving $0.9 \%$ saline solution was used to provide background fluorescence.

\section{Statistical analysis}

All data represent group means and standard errors of the mean (SEM). The experimental data in vitro and in vivo were analyzed by two-way analyses of variance (ANOVA). Individual group means were compared by the Newman-Keuls multiple-range test. GB-STAT software (Dynamic Microsystems, Inc., USA) was used for all statistical analyses. Statistical significance was defined as a two-sided $p$ value of less than 0.05 .

\section{RESULTS AND DISCUSSION}

Loading capacity of ZnPc conjugates 1-7 on $E$. coli We previously reported that many phthalocyanine pho- 
tosensitizers showed promising antibacterial effect in photodynamic antimicrobial chemotherapy (PACT) $[21,22]$. However, the loading capacities of different charged $\mathrm{ZnPc}$ conjugates to bacteria are not clear yet. For this purpose, we synthesized seven kinds of $\mathrm{ZnPc}$ photosensitizers ( $\mathrm{ZnPc}$ conjugates 1-7, Fig. 1) and investigated their loading capacities on bioluminescent $E$. coli by detecting the fluorescence intensity of these $\mathrm{ZnPc}$ conjugates@E. colivia CLSM $\left(\lambda_{\mathrm{ex}}=641 \mathrm{~nm}, \lambda_{\mathrm{em}}=690 \mathrm{~nm}\right.$, Fig. $2 \mathrm{a})$ or flow cytometer fluorescence $\left(\lambda_{\mathrm{ex}}=633 \mathrm{~nm}, \lambda_{\mathrm{em}}\right.$ $=675-725 \mathrm{~nm}$, Fig. $2 \mathrm{~b}$ ). We found that the bioluminescence produced by $E$. coli is proportional to the number of live E. coli (Fig. S1). There was no significant difference in the fluorescence intensity (Fig. 2c) or the quantum yield of singlet oxygen generation (Fig. S2) among ZnPc conjugates 1-7; however, $\mathrm{ZnPc}$ conjugate 5 (i.e., $\mathrm{ZnPc}$ IR710) exhibited the highest fluorescence intensity after 10-min incubation with E. coli, indicating that the load of ZnPc-IR710 on E. coli was much higher than that of other $\mathrm{ZnPc}$ conjugates. This may be due to the fact that $\mathrm{ZnPc}-$ IR710 has more positive charges than other counterparts, thus it is easier to be loaded on the bacterial surface.

The kinetics of ZnPc-IR710 on E. coli was detected by CLSM images, showing that the loading of ZnPc-IR710 on E. coli was stable within $8 \mathrm{~min}$ (Fig. S3). This result is consistent with our previous report that $\mathrm{ZnPc}$ photosensitizer can quickly adhere to bacteria and reach the saturation [20,21]. The fluorescence intensity of ZnPcIR710 is proportional to its concentration (Fig. S4). Then we determined the correlation between the concentration of ZnPc-IR710 and the number of ZnPc-IR710 molecules loaded within E. coli after 10-min incubation (Fig. 2d). According to the calibration curve, we estimated that each $E$. coli cell could load $\sim 1.30 \times 10^{6} \mathrm{ZnPc}$-IR710 molecules. Meanwhile, the dark toxicity of ZnPc-IR710 to E. coli was evaluated (Fig. 2e), showing no significant effect of E. coli@ZnPc-IR710 on the growth of E. coli.

\section{Cellular uptake and photocytotoxicity of E. coli@ZnPc- IR710 in vitro}

After 30-min incubation with E. coli@ZnPc-IR710 or ZnPc-IR710 (both at a concentration of $100 \mathrm{nmol} \mathrm{L}^{-1}$ ), the cellular uptake amount of E. coli@ZnPc-IR710 by MCF-7 cells was about twice that of ZnPc-IR710 under the same conditions by flow cytometry analysis (Fig. 3a, b). These results demonstrate that E. coli@ZnPc-IR710 has higher cellular uptake activity than ZnPc-IR710. Furthermore, CLSM images of MCF-7 cells (Fig. S5) showed that the fluorescence intensity of E. coli@ZnPcIR710 $\left(\lambda_{\text {ex }}=641 \mathrm{~nm}, \lambda_{\text {em }}=690 \mathrm{~nm}\right)$ was stronger than that of ZnPc-IR710 with the same concentration, confirming that the accumulation of $E$. coli promoted the cellular uptake of ZnPc-IR710. The better cellular uptake ability of bacteria-mediated system may be due to the fact that bacteria prefer to accumulate in tumor areas [23]. Compared with small molecules, such as photosensitizers, which can be pumped out of cells [24], bacteria that enter tumor cells through their flagella are difficult to expel [25]. Thus, these bacteria can be considered as natural carriers of tumor-targeting drugs. Compared with some other types of photosensitizers reported most recently, e.g., carbon nanodots [26] and lanthanides@ $\mathrm{Cu}_{2-x} \mathrm{~S}$ [27],
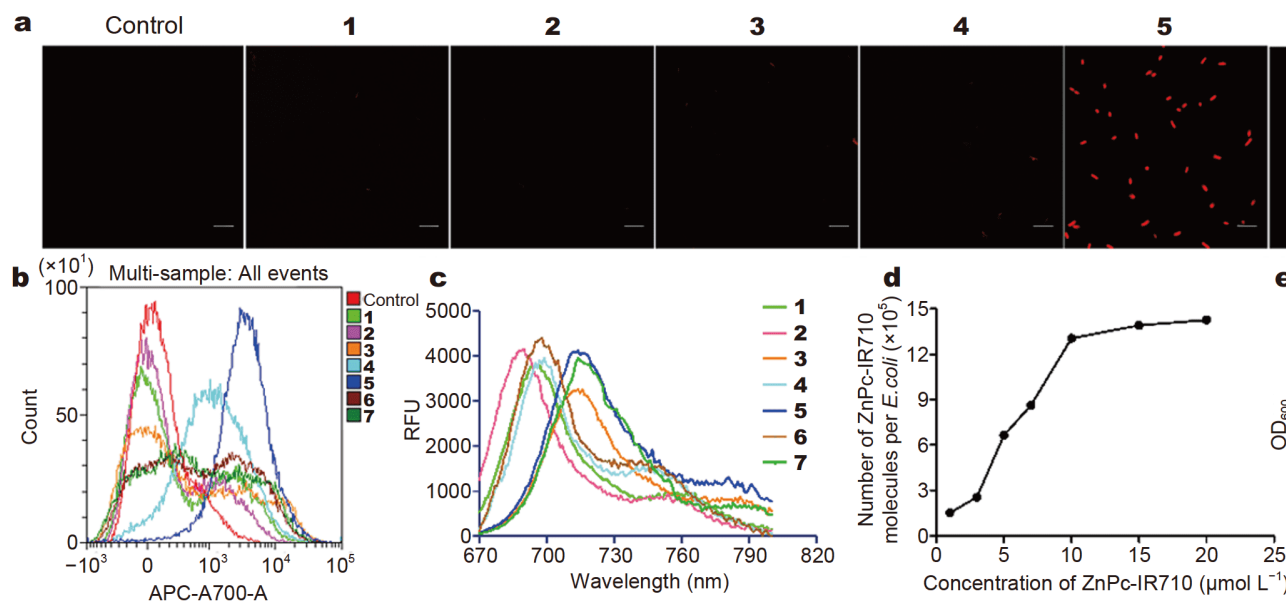

6

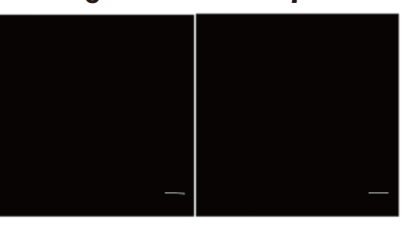

e

Figure 2 Loading capacities of ZnPc conjugates 1-7 $\left(10 \mu \mathrm{mol} \mathrm{L}^{-1}\right)$ on E. coli. CLSM (a) and flow cytometer (b) results of fluorescence intensity of $\mathrm{ZnPc}$ conjugates 1-7 after incubation with E. coli. (c) Similar fluorescence intensity (relative fluorescence units, RFU) of ZnPc conjugates 1-7 $\left(10 \mu \mathrm{mol} \mathrm{L}^{-1}\right)$ and $(\mathrm{d})$ the dose-dependent fluorescence intensity of E. coli after incubation with ZnPc-IR710. (e) The toxicity of ZnPc-IR710 $\left(10 \mu \mathrm{mol} \mathrm{L}^{-1}\right)$ for E. coli in dark. 

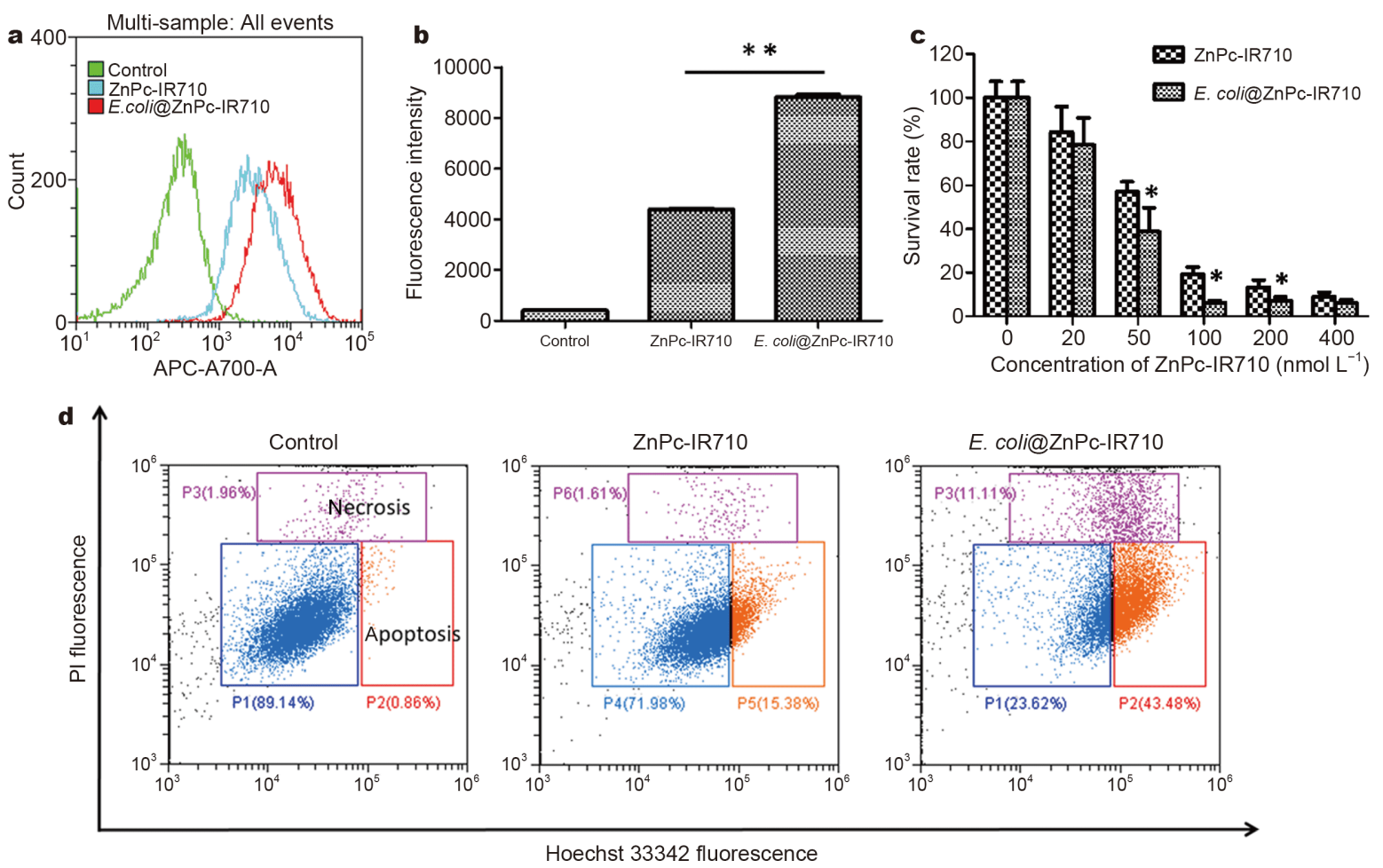

Figure 3 Phototoxicity of E. coli@ZnPc-IR710 against MCF-7 cells. (a) Flow cytometry analysis of MCF-7 cells after incubation with E. coli@ZnPcIR710 $\left(100 \mathrm{nmol} \mathrm{L}^{-1}\right)$ and ZnPc-IR710 $\left(100 \mathrm{nmol} \mathrm{L}^{-1}\right)$, respectively. (b) Quantitatively analysis of the cellular uptake. (c) Dose-dependent phototoxicity of E. coli@ZnPc-IR710 against MCF-7 cells under $9 \mathrm{~J} \mathrm{~cm}^{-2}$ irradiation. (d) Analysis of cell cycle and apoptosis of MCF-7 cells after incubation with E. coli@ZnPc-IR710 $\left(100 \mathrm{nmol} \mathrm{L}^{-1}\right)$ or ZnPc-IR710 $\left(100 \mathrm{nmol} \mathrm{L}^{-1}\right)$, respectively. Significant difference is indicated by * and ** (at $p<0.05$ and at $p<0.01$, respectively) from the respective ZnPc-IR710-treated groups.

our bacteria-mediated system shows the great potential in the treatment of solid tumors, and provides another unique strategy to enhance the antitumor efficacy of PDT by using bacterial vectors.

Next, we investigated the phototoxicity of E. coli@ZnPc-IR710 against MCF-7 cells. The photocytotoxicity of E. coli@ZnPc-IR710 was dose-dependent after incubation with MCF-7 cells for 30 min followed by irradiation under $670 \mathrm{~nm}$ at $9 \mathrm{~J} \mathrm{~cm}^{-2}$ (Fig. 3c). Compared with ZnPc-IR710, E. coli@ZnPc-IR710 showed enhanced cytotoxicity against MCF-7 cells under the same irradiation dose (e.g., survival rate of tumor cell was 39\% vs. 57\% at a concentration of $50 \mathrm{nmol} \mathrm{L}^{-1}$ ) (Fig. 3c), possibly due to the higher cellular uptake of E. coli@ZnPc-IR710 by MCF-7 cells (Fig. 3a).

To further investigate the antitumor mechanism of E. coli@ZnPc-IR710 against MCF-7 cells, Hoechst 33342/ PI double staining was applied to distinguish normal, apoptotic and dead cell populations [28,29]. The flow cytometry data showed that the apoptosis rates of MCF-7 cells in E. coli@ZnPc-IR710 group and ZnPc-IR710 group were $43.5 \%$ and $15.4 \%$, respectively (Fig. $3 \mathrm{~d}$ ). These results implied that E. coli@ZnPc-IR710 scavenges MCF-7 cells mainly through apoptotic rather than direct killing, and provided evidence for the colonization ability of E. coli.

\section{Photodynamic antitumor activity of E. coli@ZnPc-IR710 in vivo}

Before the in vivo antitumor experimental study, we injected different amounts of E. coli $\left(10^{7}\right.$ and $10^{8}$, respectively) into the mice through tail vein to determine the tolerance of mice to E. coli. The mice injected with $0.9 \%$ saline served as control. During the 10-d observation period, we did not observe any significant difference in body weight or behavior between the mice receiving E. coli or $0.9 \%$ saline (Fig. S6), indicating that mice had good tolerance to E. coli.

The antitumor effect of E. coli@ZnPc-IR710 was then evaluated on $4 \mathrm{~T} 1$ tumor-bearing mouse model. The tumor size and body weight of the mice were monitored during the 7-d PDT experiment under illumination of $30 \mathrm{~J} \mathrm{~cm}^{-2}$. We observed that the growth rates of tumor masses were significantly lower in the mice treated with $E$. coli, ZnPcIR710 or E. coli@ZnPc-IR710, compared with that of 

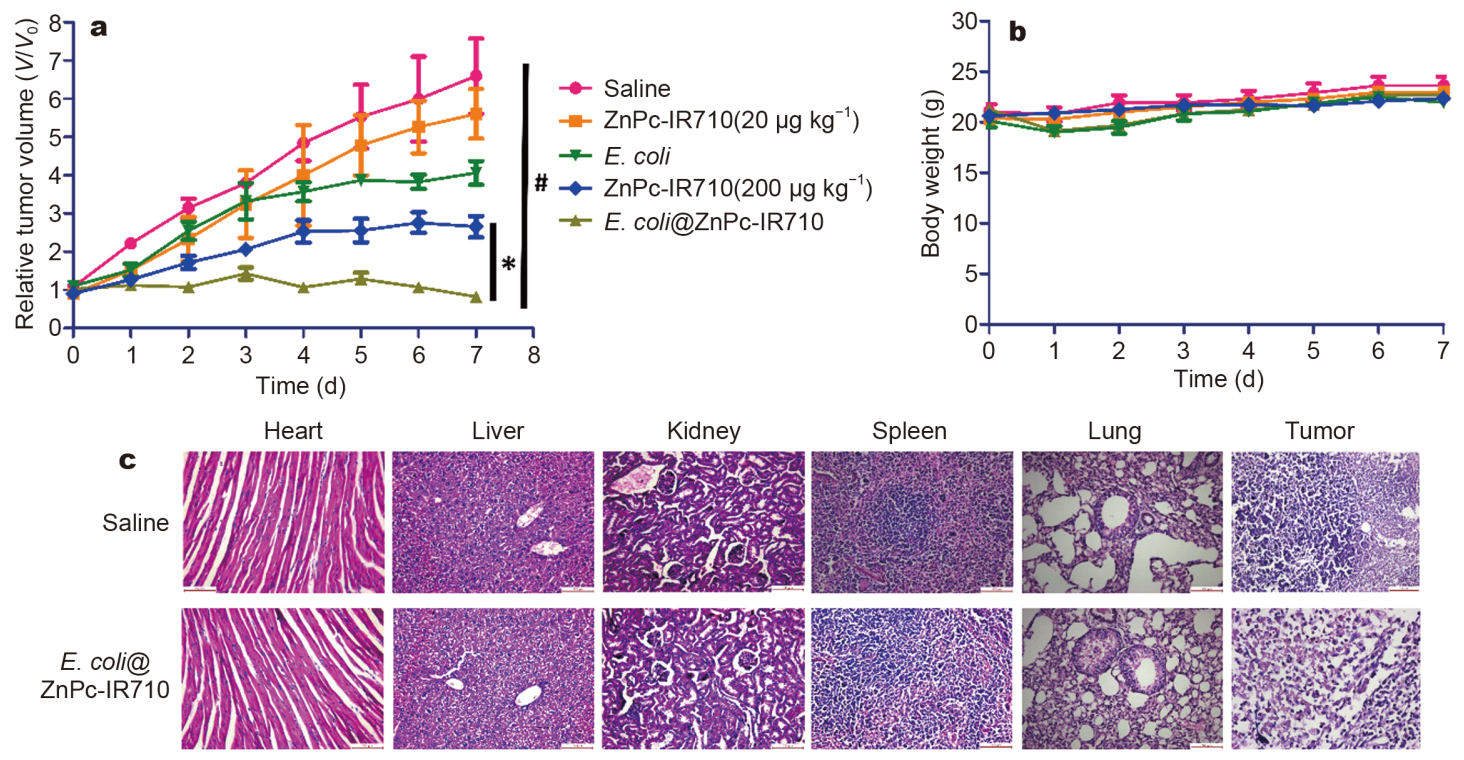

Figure 4 Antitumor effect of E. coli@ZnPc-IR710 on 4T1-bearing mice. Relative tumor volume (a) and body weight (b) of 4T1 tumor-bearing mice. (c) Histological sections of important organs (heart, liver, kidney, spleen and lung) and tumor tissues from 4T1-bearing mice after the treatment with E. coli@ZnPc-IR710 (100x). The symbol * indicates a significant difference $($ at $p<0.05)$ from the respective ZnPc-IR710-treated controls; \# indicates a significant difference $($ at $p<0.01)$ from the respective saline-treated controls.
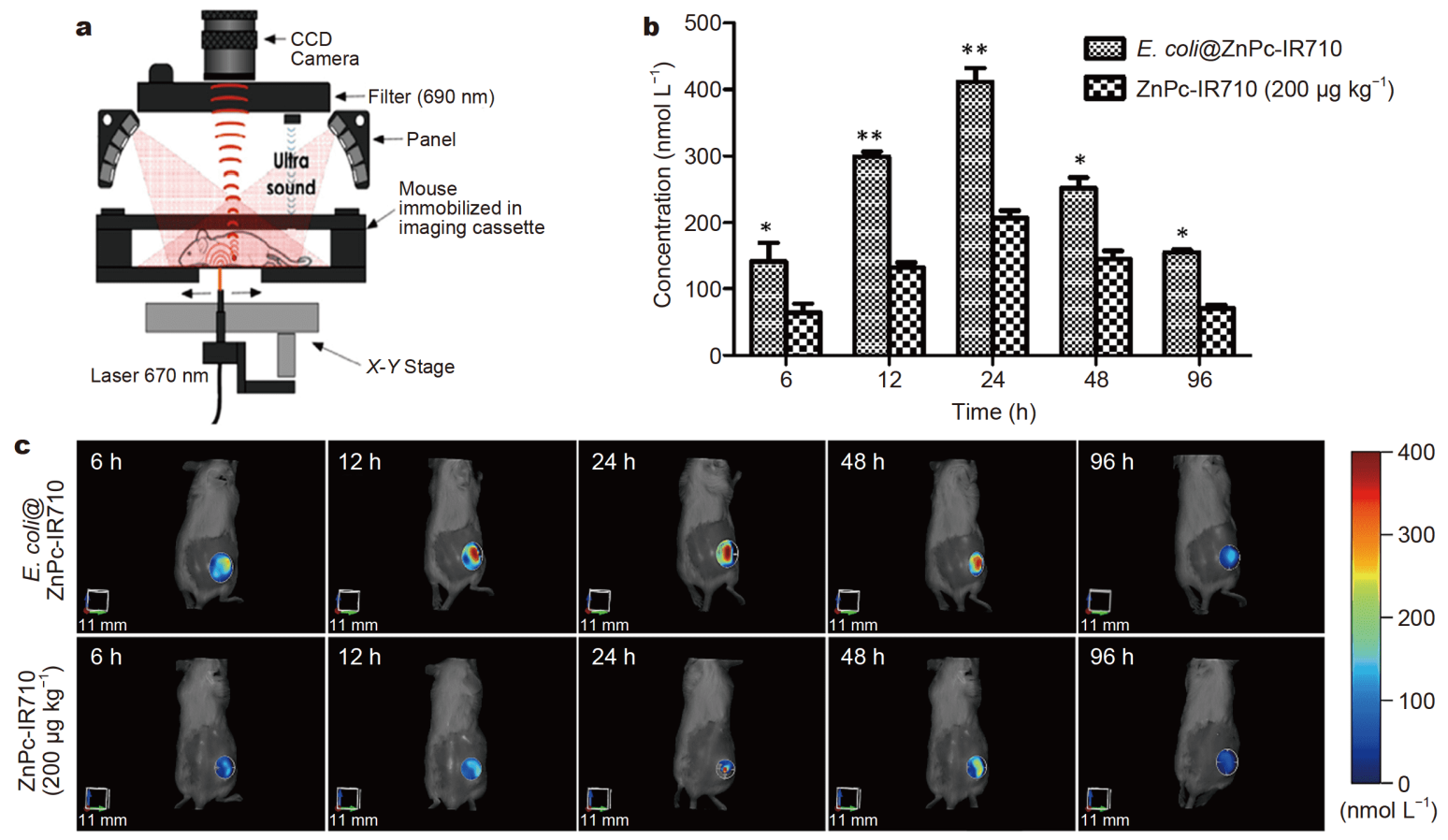

Figure 5 Pharmacokinetic study of E. coli@ZnPc-IR710 in 4T1 tumor-bearing mice. (a) Schematic diagram of the FMT imaging system. Determination of the concentration of ZnPc-IR710 in 4T1 tumor-bearing mice (b) and representative FMT images (c) at different time points after administration of E. coli@ZnPc-IR710 and ZnPc-IR710, respectively. Significant difference is indicated by * and $* *$ (at $p<0.05$ and $p<0.01$, respectively) from the respective ZnPc-IR710-treated controls. 
control group treated with 0.9\% saline (Fig. 4a). E. coli@ ZnPc-IR710 exhibited the strongest antitumor effect on $4 \mathrm{~T} 1$ tumor-bearing mice among the three treatment groups, which was consistent with our results of in vitro experiments. In addition, the weight of mice was monitored throughout the whole experiment, and no significant effect on body weight of mice was observed(Fig. 4b).

Meanwhile, the pathologic changes of important organs (heart, liver, kidney, spleen and lung) and tumor sites from the mice treated with E. coli@ZnPc-IR710 were inspected by routine tissue slice with H\&E staining. No obvious histopathological abnormality or lesion of these organs was observed (Fig. 4c), suggesting the good biosafety of E.coli@ZnPc-IR710.

\section{Pharmacokinetic study of $E$. coli@ZnPc-IR710 in tumor sites} In the present study, we used a non-invasive optical method called fluorescent molecular tomography (FMT, Fig. 5a) to determine the fluorescence intensity in tumor sites at different time points $(6,12,24,48$ and $96 \mathrm{~h})$ after injection of E. coli@ZnPc-IR710 in 4T1 tumor-bearing mice. Compared with ZnPc-IR710, E. coli@ZnPc-IR710 significantly enhanced the fluorescent signal of $\mathrm{ZnPc}$ IR710 in tumor sites at each time point (Fig. 5b), indicating that $E$. coli can significantly increase the concentration of ZnPc-IR710 by promoting its entry into tumors (Fig. 5 c). Further examination of tumor tissue by CLSM (Fig. S7) confirmed the role of bacteria in promoting the entry of ZnPc-IR710 into tumors.

\section{CONCLUSIONS}

We have developed a novel bacteria-mediated drugdelivery system in an effort to improve the efficacy of photosensitizer ZnPc-IR710 in photodynamic cancer therapy. Such a unique strategy takes advantage of the priority of non-pathogenic E. coli in tumor accumulation, and PDT with minimal invasion and toxicity. As such, each E. coli cell can carry $1.3 \times 10^{6} \mathrm{ZnPc}$ molecules, which remarkably promotes the accumulation of $\mathrm{ZnPc}$ photosensitizer in tumors and enhances the effect of eliminating tumors. Furthermore, our approach demonstrates successfully the great promise of $E$. coli as a carrier for photosensitizer to improve the therapeutic effect of PDT. These findings showcase the potential of $E$. coli-guided PDT in solid tumor therapy and provide a feasible and concise treatment for tumors.

Received 25 February 2020; accepted 18 April 2020; published online 9 July 2020

1 Jia Q, Chen M, Liu Q, et al. Ethylene glycol-mediated synthetic route for production of luminescent silicon nanorod as photodynamic therapy agent. Sci China Mater, 2017, 60: 881-891

2 Liang P, Tang H, Gu R, et al. A pH-responsive zinc(II) metalated porphyrin for enhanced photodynamic/photothermal combined cancer therapy. Sci China Mater, 2019, 62: 1199-1209

3 Han W, Zhang S, Deng R, et al. Self-assembled nanostructured photosensitizer with aggregation-induced emission for enhanced photodynamic anticancer therapy. Sci China Mater, 2020, 63: 136146

4 Flentie K, Kocher B, Gammon ST, et al. A bioluminescent transposon reporter-trap identifies tumor-specific microenvironmentinduced promoters in Salmonella for conditional bacterial-based tumor therapy. Cancer Discov, 2012, 2: 624-637

5 Stritzker J, Weibel S, Hill PJ, et al. Tumor-specific colonization, tissue distribution, and gene induction by probiotic Escherichia coli nissle 1917 in live mice. Int J Med Microbiol, 2007, 297: 151-162

6 Sasaki T, Fujimori M, Hamaji Y, et al. Genetically engineered bifidobacterium longum for tumor-targeting enzyme-prodrug therapy of autochthonous mammary tumors in rats. Cancer Sci, 2006, 97: 649-657

7 Bettegowda C, Dang LH, Abrams R, et al. Overcoming the hypoxic barrier to radiation therapy with anaerobic bacteria. Proc Natl Acad Sci USA, 2003, 100: 15083-15088

8 Neri D, Supuran CT. Interfering with $\mathrm{pH}$ regulation in tumours as a therapeutic strategy. Nat Rev Drug Discov, 2011, 10: 767-777

9 Khong HT, Restifo NP. Natural selection of tumor variants in the generation of "tumor escape" phenotypes. Nat Immunol, 2002, 3: 999-1005

10 Webb BA, Chimenti M, Jacobson MP, et al. Dysregulated pH: A perfect storm for cancer progression. Nat Rev Cancer, 2011, 11: 671-677

11 Yazawa K, Fujimori M, Nakamura $\mathrm{T}$, et al. Bifidobacterium longum as a delivery system for gene therapy of chemically induced rat mammary tumors. Breast Cancer Res Treat, 2001, 66: $165-170$

12 Zheng DW, Chen Y, Li ZH, et al. Optically-controlled bacterial metabolite for cancer therapy. Nat Commun, 2018, 9: 1680

13 Fan JX, Li ZH, Liu XH, et al. Bacteria-mediated tumor therapy utilizing photothermally-controlled TNF- $\alpha$ expression via oral administration. Nano Lett, 2018, 18: 2373-2380

14 Jia LJ, Xu HM, Ma DY, et al. Enhanced therapeutic effect by combination of tumor-targeting salmonella and endostatin in murine melanoma model. Cancer Biol Ther, 2005, 4: 840-845

15 Low KB, Ittensohn M, Le T, et al. Lipid a mutant salmonella with suppressed virulence and TNFa induction retain tumor-targeting in vivo. Nat Biotechnol, 1999, 17: 37-41

16 Zhang HY, Man JH, Liang B, et al. Tumor-targeted delivery of biologically active trail protein. Cancer Gene Ther, 2010, 17: 334343

17 Cheng $\mathrm{CM}$, Lu YL, Chuang $\mathrm{KH}$, et al. Tumor-targeting prodrugactivating bacteria for cancer therapy. Cancer Gene Ther, 2008, 15: 393-401

18 Chen J, Chen N, Huang J, et al. Derivatizable phthalocyanine with single carboxyl group: Synthesis and purification. Inorg Chem Commun, 2006, 9: 313-315

19 Liu JY, Jiang XJ, Fong WP, et al. Highly photocytotoxic 1,4-dipegylated zinc(II) phthalocyanines. Effects of the chain length on the in vitro photodynamic activities. Org Biomol Chem, 2008, 6: 4560

20 Chen Z, Zhou S, Chen J, et al. An effective zinc phthalocyanine 
derivative for photodynamic antimicrobial chemotherapy. J Lumin, 2014, 152: 103-107

21 Zhang Y, Zheng K, Chen Z, et al. Rapid killing of bacteria by a new type of photosensitizer. Appl Microbiol Biotechnol, 2017, 101: 4691-4700

22 Zhang Y, Huang P, Wang D, et al. Near-infrared-triggered antibacterial and antifungal photodynamic therapy based on lanthanide-doped upconversion nanoparticles. Nanoscale, 2018, 10: 15485-15495

23 Forbes NS. Engineering the perfect (bacterial) cancer therapy. Nat Rev Cancer, 2010, 10: 785-794

24 Boumahdi S, de Sauvage FJ. The great escape: tumour cell plasticity in resistance to targeted therapy. Nat Rev Drug Discov, 2020, 19: $39-56$

25 Xie S, Zhao L, Song X, et al. Doxorubicin-conjugated Escherichia coli nissle 1917 swimmers to achieve tumor targeting and responsive drug release. J Control Release, 2017, 268: 390-399

26 Li Y, Bai G, Zeng S, et al. Theranostic carbon dots with innovative NIR-II emission for in vivo renal-excreted optical imaging and photothermal therapy. ACS Appl Mater Interfaces, 2019, 11: 47374744

27 Jiang M, Liu H, Zeng S, et al. A general in situ growth strategy of designing theranostic $\mathrm{NaLnF}_{4} @ \mathrm{Cu}_{2-x} \mathrm{~S}$ nanoplatform for in vivo NIR-II optical imaging beyond $1500 \mathrm{~nm}$ and photothermal therapy. Adv Therap, 2019, 2: 1800153

28 Yi $\mathrm{P}$, Chen $\mathrm{G}$, Zhang $\mathrm{H}$, et al. Magnetic resonance imaging of $\mathrm{Fe}_{3} \mathrm{O}_{4} @ \mathrm{SiO}_{2}$-labeled human mesenchymal stem cells in mice at 11.7 T. Biomaterials, 2013, 34: 3010-3019

29 Tang S, Hu J, Meng Q, et al. Daidzein induced apoptosis via downregulation of $\mathrm{Bcl}-2 / \mathrm{Bax}$ and triggering of the mitochondrial pathway in BGC-823 cells. Cell Biochem Biophys, 2013, 65: 197-202

Acknowledgements This work was supported by the National Natural Science Foundation of China $(81572944,21471033,21877113$ and 81971983), the CAS/SAFEA International Partnership Program for Creative Research Teams, the High-Level Entrepreneurship and Innovation Talents Projects in Fujian Province (2018-8-1), and the FJIRSM\&IUE Joint Research Fund (RHZX-2018-004).

Author contributions Dai $\mathrm{T}$ and Chen $\mathrm{Z}$ conceived and designed the project. Ye F, Hu P and Chen J designed and synthesized zinc phthalocyanine conjugates 1-5. Dai T and Wang $\mathrm{H}$ performed the experiments. Dai T, Zhang L and Pan X analyzed the data. Chen J, Huang Y, Pan X and Huang $M$ provided the technical support. Chen $Z$ and Dai $T$ finished the writing.

Conflict of interest The authors declare that they have no conflict of interest.

Supplementary information Experimental details and supporting data are available in the online version of the paper.

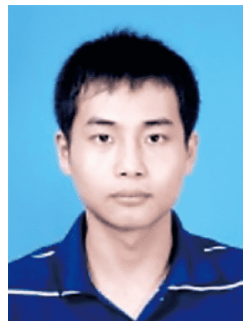

Tao Dai received his BSc degree in biotechnology from Anhui Normal University in 2014 Currently he is a Master student in Prof. Zhuo Chen's group at Fujian Institute of Research on the Structure of Matter (FJIRSM), Chinese Academy of Sciences. His current research focuses on the targeted drug delivery in tumor therapy.

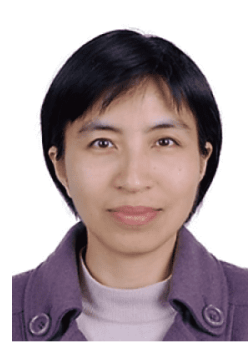

Zhuo Chen received her PhD from FJIRSM, Chinese Academy of Sciences, following her MD degree from Fujian Medical University and her BSc degree from Chinese Pharmaceutical University. From 2001 to 2008, she worked on molecular pharmacology \& therapeutics in Loyola University Chicago as a Research Associate. Her research focuses on tumor detection and targeted photodynamic therapy, including design and synthesis of new anticancer drug entities and evaluation of their pharmacological effects through experiments in cells and animals.

\section{一种基于大肠杆菌驱动药物递送系统增强光动力 治疗的肿瘤靶向策略}

戴涛 ${ }^{1}$, 叶发荣 ${ }^{2}$, 胡萍 ${ }^{1,2}$, 潘小宏 ${ }^{1}$, 陈锦灿 ${ }^{1,5}$, 黄云梅 ${ }^{3}$, 王欢欢 ${ }^{1,5}$,

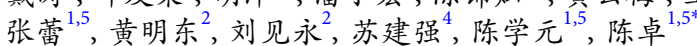

摘要 大肠杆菌(简称E. coli) DH $5 a$ 被认为是具有肿瘤定植能力的 非致病菌, 然而这种细菌驱动的药物传递系统能否提高肿瘤治疗 的靶向性却鲜有报道. 本文中, 我们通过制备一系列带有不同电荷 数的酞菁锌 $(\mathrm{ZnPc})$ 光敏剂, 研究了它们在大肠杆菌上的静电黏附特 性, 并借助流式细胞仪检测了其荧光强度. 在所研究的 ZnPc光敏剂 中，带有 4 个正电荷的酞菁光敏剂ZnPc-IR710对E. coli具有最高的 载药量和最佳的荧光成像性能. 实验结果表明, 与没有 E. coli的 ZnPc-IR710比较, E. coli@ZnPc-IR710对人乳腺癌MCF-7细胞的细 胞毒性显著增强(同样条件下, 该肿瘤细胞的存活率分别为 $39 \%$ (50 nmol L ${ }^{-1}$ E. coli@ZnPc-IR710)和57\% (ZnPc-IR710). 此外, 在体 药理实验研究表明, E. coli@ZnPc-IR710对小鼠4T1皮下乳腺肿瘤的 生长具有明显抑制作用, 可完全抑制肿瘤生长. 这些实验结果显示 了利用细菌作为药物驱动传递系统在光动力疗法治疗实体肿瘤中 的巨大潜力, 同时也为增强光动力疗法的靶向抗肿瘤作用提供了 独特策略. 